pÿUse of fertility drugs in early onset female cancer survivors A Finnish register-based study on 8,929 survivors

\title{
Melin, Johanna
}

2020-02-01

Melin , J , Madanat-Harjuoja , L , Hirvonen , E , Seppä , K, Malila , N , Pitkäniemi , J , Gissler , M \& Tiitinen , A 2020 , ' Use of fertility drugs in early onset female cancer survivors pÿ A Finnish register-based study on 8,929 survivors ' , International Journal of Cancer , vol. 146 , no. 3 , pp. $829-838$. https://doi.org/10.1002/ijc.32346

http://hdl.handle.net/10138/314132

https://doi.org/10.1002/ijc.32346

unspecified

acceptedVersion

Downloaded from Helda, University of Helsinki institutional repository.

This is an electronic reprint of the original article.

This reprint may differ from the original in pagination and typographic detail.

Please cite the original version. 


\section{Use of fertility drugs in early onset female cancer survivors -A Finnish register-}

\section{based study on 8,929 survivors}

Short title: Fertility Treatments in Female Cancer Survivors

Corresponding author: Johanna Melin, Finnish Cancer Registry, Unioninkatu 22, 00130 Helsinki, Finland.

Electronic address: Johanna.Melin@cancer.fi. Tel. +358 505382526

2

Key words: Female cancer survivors, infertility, fertility treatments, ART

Abbreviations used: OI, Ovulation Induction; ART, Assisted Reproductive Technology; IVF, In vitro Fertilization; GnRH, Gonadotropin Releasing Hormone; PIC, Personal Identity Code; FCR, Finnish Cancer Register; CPR, Central Population Register; MBR, Medical Birth Register; RPM, Reimbursement Register for Prescribed Medicines; IRR, Incidence rate ratio; $\mathrm{Cl}$, Confidence Interval; ICCC3, International Classification of Childhood Cancer; Pyrs, Person years 1

Conflict of interest: No conflicts of interest exist. (1)

(1)

(

Article Category: Cancer Epidemiology

Novelty and Impact: A previous study showed that early onset female cancer survivors were less likely to receive fertility treatments compared to siblings. However, according to our study, female cancer survivors are more likely to use fertility drugs compared to siblings in the last decade. The use of assisted reproductive technology was significantly increased, whereas the use of ovulation induction in cancer survivors was similar to that of siblings.

This article has been accepted for publication and undergone full peer review but has not been through the copyediting, typesetting, pagination and proofreading process, which may lead to differences between this version and the Version of Record. Please cite this article as doi: $10.1002 / \mathrm{ijc} .32346$ 


\section{Use of fertility drugs in early onset female cancer survivors -A Finnish register- based study on 8,929 survivors}

Johanna Melin MD ${ }^{1,2}$, Laura Madanat-Harjuoja MD, PhD ${ }^{1}$, Elli Hirvonen MSc ${ }^{1}$, Karri Seppä PhD ${ }^{1}$, Nea Malila MD, PhD ${ }^{1}$, Janne Pitkäniemi PhD ${ }^{1,3}$, Mika Gissler PhD ${ }^{4,5}$, Aila Tiitinen MD, PhD ${ }^{6}$

(2)

$>^{1} \mathrm{~F}$

${ }^{1}$ Finnish Cancer Registry, Institute for Statistical and Epidemiological Cancer Research, Helsinki, Finland 8

${ }^{2}$ Department of Obstetrics and Gynecology, Kymenlaakso Central Hospital, Kotka, Finland

${ }^{3}$ Department of Public Health, School of Medicine, University of Helsinki, Helsinki, Finland

${ }^{4}$ National Institute for Health and Welfare, Information Services Department, Helsinki, Finland

${ }^{5}$ Karolinska Institute, Department of Neurobiology, Care Science and Society, Stockholm, Sweden (1) 6 1

${ }^{6}$ Department of Obstetrics and Gynecology, University of Helsinki, Helsinki University Hospital, Helsinki,

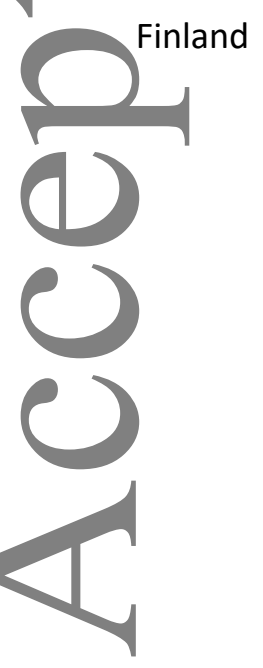




\section{Abstract}

Advances in multimodality cancer treatments have increased the risk of long-term complications in early onset cancer survivors. For female cancer survivors these include diminished reproductive function, often resulting in a narrowed fertile window. The aim of this study was to evaluate the use of fertility treatments in cancer survivors (aged 0-39 years at diagnosis) compared to siblings. Data from Finnish registers on cancer, birth and prescribed medications were merged to identify 8,929 survivors and 9,495 siblings without previous deliveries. Fertility drug purchases from 1993 to 2012 at the age of 16-41 years were included. A Poisson regression model was used to estimate incidence rate ratios (IRR) for the use of fertility drugs, adjusting for age and calendar time at fertility drug purchase. Fertility treatments were more common in survivors compared to siblings, as $6.1 \%$ of survivors compared to $3.8 \%$ of siblings had bought fertility drugs (IRR 1.43, 95\% confidence interval [CI] 1.25-1.65). A sub-classification of fertility treatments into ovulation inductions and assisted reproductive technology (ART), showed increased use of ART (IRR 1 $2.41,95 \% \mathrm{Cl}$ 1.97-2.96), whereas the use of ovulation induction was similar in survivors and siblings. Analyses by calendar time periods showed the use of ART to be significantly higher in the most recent decade, from 2003 onwards. We conclude that cancer survivors have an increased risk for subfertility, which is why fertility counselling is important. However, our results mirror a more active approach among clinicians towards fertility treatments in cancer survivors during the most recent years. 


\section{Introduction}

The population of early onset cancer survivors has been increasing over the past four decades, with 5-year survival rates reaching $80 \%$ in Finland as well as in Western Europe. ${ }^{1,2}{ }^{2}$ As survival rates increase, so does the risk for chronic health conditions due to previous exposure to toxic anti-cancer treatments. Of these

late effects, gonadal dysfunction and reduced fertility have been reported as major concerns among cancer survivors. ${ }^{4} \mathrm{~A}$ previous study ${ }^{5}$ found that cancer treatments, especially abdominal radiation and chemotherapy with alkylating agents, may damage the ovaries, leading to diminished ovarian reserve, a narrowed fertile window and even premature ovarian insufficiency.

1

8

(n)

Many studies have shown reduced parenthood and pregnancy rates in cancer survivors compared to siblings or the general population. ${ }^{6-9}$ There are, however, only a few studies focusing on infertility and even less on the use of fertility treatments among cancer survivors. ${ }^{10,11}$ One of them reported an increased risk for or clinical infertility (defined as the inability to conceive after at least one year of unprotected intercourse C during the fertile phase of the menstrual cycle) among survivors compared to siblings. ${ }^{10}$ In this study, Survivors were as likely as their siblings to seek medical help for their infertility, but less likely to receive fertility treatments. ${ }^{10}$ However, a previous study with the Finnish cohort ${ }^{11}$ found that cancer survivors giving birth were more likely to undergo fertility treatments than their siblings were. Time elapsed from (1) cancer treatment played a central role, with increasing use of fertility treatments over time.

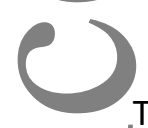

The aim of our study was to investigate whether female cancer survivors (diagnosed with cancer at the age of 0-39 years) receive more fertility treatments or fertility preservation than their siblings do, and if type of used fertility drug differed from that of siblings by the use of various Finnish register databases (FCR, CPR, MBR and RPM). 


\section{Materials and Methods}

In this retrospective, register-based study, we used data from four different national population-based registers: The Finnish Cancer Registry (FCR), the Central Population Register (CPR), the Medical Birth Register (MBR) and the Reimbursement Register for Prescribed Medicines (RPM). The registers were linked using the personal identity code (PIC) given to all Finnish citizens and residents.

\section{Registers}

The FCR is a population based national register on incident cancer cases since 1953 . The coverage of the JFCR is high, $96 \%$ for solid tumors and $86 \%$ for hematological malignancies. Of all cancers, $93 \%$ were morphologically verified. ${ }^{12}$ Data on cancer patients include: the PIC, details on cancer (site, histology and malignancy), treatment data (radiotherapy, chemotherapy, surgery and hormonal) and month as well as year of diagnosis. Clinical information is mainly provided by the treating physician and the histological data is notified by the pathology department. Childhood cancer diagnoses are categorized using the International Classification of Childhood Cancer (ICCC3). ${ }^{13}$ $+$

The CPR was founded in 1969 and computerized registration began in 1971. The register is national, covering all citizens and residents in Finland and includes data on residential history and death. Individuals ( ) born in 1955 or thereafter can be linked reliably to their parents, siblings and offspring.

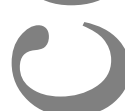

The MBR was established in 1987 and contains detailed information on all mothers giving birth as well as their children. Data is collected at the delivery hospital, using the maternity records of the mothers and includes information on all live births and stillbirths with a birth weight of more than $500 \mathrm{~g}$ or a pregnancy lasting for at least 22 weeks are registered. Data on less than $0.1 \%$ of newborns are missing in the Medical Birth Register. ${ }^{14}$ 
The RPM has registered all purchased drugs since 1993. All refundable medications prescribed by public as well as by private health care providers are registered. Data include the purchase date of the prescription drug, the price of the medication and package size. All the drugs have been listed according to Anatomical Therapeutic Chemical codes (ATC codes) categories released by the World Health Organization. A study ${ }^{15}$ comparing data from RPM and aggregated statistics of in vitro fertilization (IVF) in Finland, found the coverage of this register to be good. ${ }^{15}$

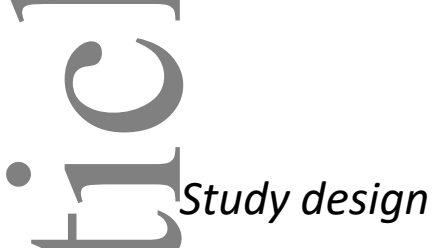

From the FCR, we identified 23,125 female cancer patients (hereafter referred to as survivors) diagnosed with a malignant neoplasm or benign brain tumor under the age of 40 years between January 1953 and December 2012 (Figure 1). Female siblings of survivors were identified from the CPR by maternal link using the PICs. We identified 20,542 female siblings of survivors. To eliminate possible influences of pregnancy history on the endpoint studied, we only included women without biological children before the time-point (1) of fertility drug purchase. By linkage to the MBR, we could exclude women who were multiparous and by linking to the RPM, we could exclude women who had delivered before fertility drug purchase. This way, we identified 16,640 survivors and 18,184 siblings without deliveries prior to drug purchase between 1993 and 2012.

C We excluded all women who were not in the age range of 16-41 years between 1993 and 2012 (when information on fertility drug purchase was available), and were left with 8,929 survivors and their 9,495 siblings. Censoring events before drug purchase (Figure 1) included a pregnancy of more than 22 weeks or birth (a notification in the MBR), death, emigration, notification of aromatase inhibitor medication as a long-term anti-cancer treatment (registered in the RPM) or cancer diagnosis in a sibling. After these exclusions, the follow-up time was 48,638 person years for survivors and 56,102 person years for siblings. 


\section{Statistical analysis}

In this study, use of fertility treatments in cancer survivors and their siblings, was based on information on fertility drug purchase. We estimated incidence rates of fertility treatments (the number of events per 10,000 person-years) by time period (1993-1997, 1998-2002, 2003-2007 and 2008-2012) and attained age

(16-19, 20-24, 25-29, 30-34 and 35-41 years). Within each stratum of time period and attained age, we estimated the conditional probability of receiving a fertility treatment during a given age interval, given that the woman had not received fertility treatments before. We estimated incidence rate ratios (IRR) of fertility treatments in survivors compared to siblings, with 95\% confidence intervals (CI) using Poisson regression models, adjusting for time period and attained age. A single, overall IRR, assuming no heterogeneity, was estimated. In addition, we fitted models that allowed heterogeneity in IRR by time period and age at fertility drug purchase and reported relative excess risk compared to a baseline IRR (age 20-24 years, period 1993-1997). The statistical significance of the heterogeneity was tested using the likelihood ratio test and the level of statistical significance was set at $p<0.05$. We plotted smoothed incidence rates of the different fertility treatments by age and time period, using generalized additive models. ${ }^{16}$

\section{All analyses were conducted using R version 3.5.1.}

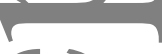

()

\section{Ethics}

The National Institute for Health and Welfare (Dnr THL/1/5.05.00/2014) and the Social Insurance Institution (Dnr KELA/69/522/2014) approved this study protocol and granted a permit for the study. The permit process included an ethical evaluation.

\section{Results}

The descriptive and treatment characteristics of female cancer survivors are presented in Table 1. Most survivors in our data (62.7\%) were diagnosed with cancer between 1993 and 2012 and the majority in 
adulthood at the age of 25-39 years (59.1\%). Childhood cancer survivors (0-14 years when diagnosed) accounted for $15.7 \%$ of all survivors, and adolescents and young adults (15-24 years when diagnosed) for 25.2\%. The largest diagnostic group based on the ICCC3 classification consisted of carcinomas and other malignant epithelial neoplasms (50.6\%). This group included breast cancers (15.7\%), cancer of the thyroid gland (11.0\%) and melanomas of the skin (7.9\%) among others. The second largest diagnostic group consisted of lymphomas (13.0\%), followed by tumors of the central nervous system (10.9\%) and leukemia (7.7\%).

In our data (Table 1), most fertility treatments (65.3\%) were given to adult cancer survivors and most often (44.7\%) within three years of cancer diagnosis. Also most ART cycles were given to survivors who had been diagnosed with cancer within three years (60.1\%). Of all cancer survivors, 49 (9.1\%) were prescribed fertility drugs within 2 months after cancer diagnosis. Exploring the different cancer types, breast cancer survivors received the largest proportion of the fertility treatments (40.9\%), the majority of ART cycles $(55.2 \%)$ and a much smaller proportion of OI (12.7\%).

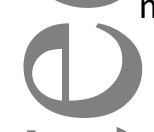

$+1$

Of all cancer survivors, 541 (6.1\%) were prescribed fertility drugs (Table 2). A majority, $64.3 \%$ of these ility treatments, were ART. Of all siblings, $358(3.8 \%)$ received fertility treatments, of which $39.4 \%$ were ART. As shown in Table 2, there was an overall increased use of fertility treatments in cancer survivors compared to siblings (IRR $1.43,95 \% \mathrm{Cl} 1.25-1.65$ ). A sub-classification of fertility treatments showed that the use of ART was significantly higher in cancer survivors (IRR $2.41,95 \% \mathrm{Cl} 1.97-2.96$ ) compared to siblings, whereas there was no increase in the use of OI (IRR $0.83,95 \% \mathrm{Cl} 0.68-1.02)$.

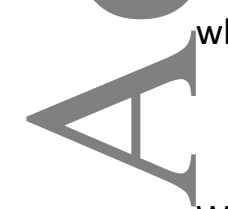

We observed statistically significant heterogeneity in IRR between age-groups and time periods in any fertility treatment and ART (Table 2). Compared to the first period, IRR between survivors and siblings was significantly larger in the most recent time periods. For example, IRR for use of ART was 3.39 -fold $(95 \% \mathrm{Cl}$ 1.79-6.41) in 2003-2007 compared to that in 1993-1997. Regarding the effect of age, IRR for the use of ART 
was significantly smaller among $30-34$ year old women (0.41-fold, $95 \% \mathrm{Cl} 0.17-0.97)$ as well as $35-41$ year old women (0.35-fold, $95 \% \mathrm{Cl} 0.15-0.82)$ compared to those aged $20-24$ years (Table 2$)$. The IRR for OI was not significantly affected by time period or age.

(1)

n Figure 2, the incidence rates of $\mathrm{OI}$ and ART are plotted against age at fertility drug purchase in different time periods. The incidence rates are given in table 3 (OI) and 4 (ART). In 1993-1997, the incidence rates for both $\mathrm{OI}$ and ART in survivors and siblings were quite similar. In OI, the same pattern could also be observed in 1998-2002. With regard to ART in 1998 to 2002, an increasing though non-significant gap in the incidence rates of survivors and siblings was visible. ART was more common among survivors than siblings in all agegroups. The biggest difference in incidence rate during this time period was among women 25-29 years of age, as the incidence rate for survivors was $47 / 10,000$ person years (Pyrs) compared to $26 / 10,000$ Pyrs in siblings. During 2003 to 2007, the difference in incidence rates for ART became significant and was at its highest. For a survivor aged 25-29 years the incidence rate of receiving ART was 85/10,000 Pyrs whereas it was $11 / 10,000$ Pyrs among siblings. At the age of $30-34$ years the incidence rate was $150 / 10,000$ Pyrs among survivors and 60/10,000 Pyrs among siblings. The incidence rates for OI were quite similar in 20032007 and 2008-2012, siblings received more OI, especially in the higher age groups. During the most recent e period of 2008-2012, the difference in incidence rates decreased for ART, still remaining significantly higher in survivors compared to siblings in all age-groups.

$\circlearrowright$

\section{Discussion}

In our study, cancer survivors had a more than 1.4-fold use of overall fertility treatments compared to siblings. A sub-classification of overall fertility treatments into OI and ART revealed that this could be explained by ART, for which the use was more than 2.4 -fold in survivors compared to siblings. Concerning Ol, there was no difference between survivors and siblings and the risk was unaffected by age or time period of fertility drug purchase. 
A report from the Childhood Cancer Survivor Study ${ }^{10}$ found a $48 \%$ increased risk for clinical infertility in cancer survivors, but on the contrary to our result cancer survivors were less likely than their siblings to be prescribed drugs for fertility treatment ( $R R 0.57,95 \% \mathrm{Cl} 0.46-0.70)$. This study, however, evaluated fertility treatments between 1992 and 2004 and does not necessary reflect the situation in recent years.

Furthermore, this study only included women younger than 21 years of age at cancer diagnosis whereas Jur study cohort includes women aged up to 40 years at cancer diagnosis.

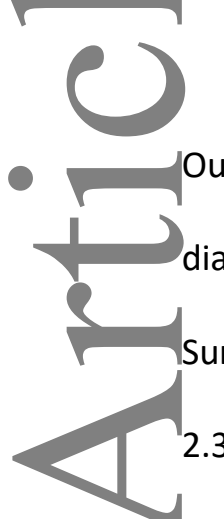

Our previous study found that Finnish female cancer survivors giving birth (aged 0-34 years at cancer diagnosis) had an almost two-fold risk for fertility treatments, during 2004-2013, compared to siblings. ${ }^{11}$ Survivors diagnosed as adults (at ages 25-34 years) had the highest odds for use of fertility treatments (OR $2.31,95 \% \mathrm{Cl} 1.01-5.32$ ) whereas childhood cancer survivors (aged 0-14 years) had the lowest risk compared to siblings. Time elapsed from cancer treatment played a central role, increasing the need for fertility U. treatments compared to siblings, suggesting that cancer therapies might lead to diminished ovarian reserve. This finding is supported by another recent study ${ }^{17}$, in which the likelihood of a live birth was reduced after ART with autologous oocytes in cancer survivors. However, after ART with donor oocytes, the live birth rate was similar to that of siblings.

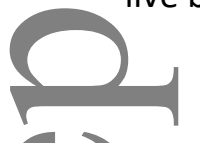

To our knowledge, there are no published reports in the literature that uses a nationwide drug prescription register to explore use of fertility treatments in cancer survivors and their siblings. Studying fertility treatments in cancer survivors is of clinical importance, since they reflect possible gonadal damage caused by cancer treatments. They also mirror the attitudes of the treating physicians towards fertility treatments and fertility preservation in cancer survivors. In our study, $9.1 \%$ of all cancer survivors were prescribed fertility drugs within 2 months after cancer diagnosis, indicating that they were possibly fertility preservations. According to recent clinical guidelines, the possibility of infertility should be discussed with all cancer patients or their parents as early as possible after cancer diagnosis and when needed, fertility preservation should be considered. ${ }^{18}$ Pregnancy after cancer is generally considered safe and should not be 
discouraged. ${ }^{19}$ When it comes to recommended time between end of cancer treatment and pregnancy, there is no standard time interval. Instead, individual timing, taking into account cancer type, extent of disease and risk of relapse is recommended. ${ }^{20}$ Also the age of the woman and her ovarian reserve should be taken into account. ${ }^{20}$ Regarding fertility treatments in female cancer survivors, one study on ART after breast cancer ${ }^{21}$ found no increased risk of relapse compared to survivors without fertility treatment. In this study, however, the majority of treatments consisted of oocyte donation and ovulation induction, which are procedures requiring no or low exposure to hormonal stimulation. In our study, $60 \%$ of all ART were

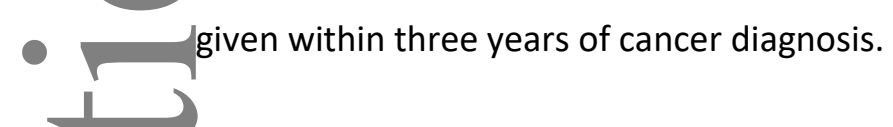
well as antral follicle count by ultra-sound, is used. ${ }^{22,23}$ Generally, OI is considered the first line treatment in ovulatory disorders and unexplained infertility, as it is less expensive, less invasive and easier to perform than ART. This was also the case for most siblings who received fertility treatments in our study. However, in survivors, the most common fertility treatment was ART, probably due to higher success rates than OI, especially when ovarian reserve is reduced. It is well known that cancer treatments, especially abdominal radiation and chemotherapy with alkylating agents, may damage the ovaries, leading to a narrowed fertile e window in cancer survivors. ${ }^{5,11}$ This probably explains the increased use of more advanced fertility treatments (ART) at the cost of easier, less invasive fertility treatments (OI) in cancer survivors. U)

The main strength of our study is outcome ascertainment by use of a national drug prescription register to obtain outcome data, thus eliminating possible recall bias. Drugs classified as fertility drugs in our study are rarely used for other conditions. In our study, cancer survivors with a notification in the RPM of aromatase inhibitor use as an anti-cancer treatment (sometimes used in breast cancer) were censored from the study. Reliable population-based registers allowed identification of both cancer and sibling cohorts with no loss to follow-up, thus eliminating participation and selection bias. By comparing survivors with siblings, we were also able to adjust for familial factors that might interfere with the outcome studied. 


\section{Conflicts of interests}

No conflicts of interests exist.

\section{References}

(1) Madanat-Harjuoja LM, Pokhrel A, Kivivuori SM, Saarinen-Pihkala UM. Childhood cancer survival in Finland (1953-2010): a nation-wide population-based study. Int J Cancer. 2014 Nov 1; 135(9):2129-34.

(2) Gatta G, Botta L, Rossi S, Aareleid T, Bielska-Lasota M, Clavel J, Dimitrova N, Jakab Z, Kaatsch P, Lacour B, Mallone S, Marcos-Gragera R, Minicozzi P, Sanchez-Perez MJ, Sant M, Santaquilani M, Stiller C, Tavilla A, TramaA, Visser O, Peris-bonet R. Childhood cancer survival in Europe 1999-2007: results of EUROCARE-5-a population based study. Lancet Oncol. 2014 Jan; 15(1):35-47.

(3) Trama A, Botta L, Foschi R, Ferrari A, Stiller C, Desandes E, Maule MM, Merlett F, Gatta G. Survival of European adolescents and young adults diagnosed with cancer in 2000-07: population-based data from EUROCARE-5. Lancet Oncol 2016 Jul; 17(7):896-906

(4) Peate M, Meiser B, Hickey M, Friedlander M. The fertility-related concerns, needs and preferences of younger women with breast cancer: a systematic review. Breast Cancer Res Treat 2009; 116: 215-223.

(5) Anderson R, Mitchell R, Kelsey T, Spears N, Telfer E, Wallace H. Cancer treatment and gonadal function: experimental and established strategies for fertility preservation in children and young adults. Lancet Diabetes Endocrinol. 2015 Jul;3(7):556-67

(6) Madanat L, Malila N, Dyba T, Hakulinen T, Sankila R, Boice J, Lähteenmäki P. Probability of parenthood after early onset cancer: A population-based study. Int J Cancer. 2008 Dec 15; 123(12):2891-2898 (7) Green D, Kawashima T, Stovall M, Leisenring W, Sklar C, Mertens A, Donaldson S, Byrne J, Robinson L. Fertility of female survivors of childhood cancer: A report from the Childhood cancer survivor study. J Clin Oncol 2009 Jun; 27(16):2677-2685

(8) Stensheim H, Cvancarova M, Moller B, Fosså S. Pregnancy after adolescent and adult cancer: A population-based matched cohort study. Int J. Cancer 2011 Sep 1;129(5):1225-36 
(9) Anderson R, Brewster D, Wood R, Nowell S, Fischbacher C, Kelsey T, Wallace H. The impact of cancer on subsequent chance of pregnancy: a population-based analysis. Hum Reprod. 2018 Jul; 33(7):1281-1290

(10) Barton S, Najita J, Ginsburg E, Leisenring W, Stovall M, Weathers R, Sklar C, Robinson L, Diller L. Infertility, infertility treatment, and achievement of pregnancy in female survivors of childhood cancer: a report from the Childhood Cancer Survivor Study cohort. Lancet Oncol 2013; 14: 873-881.

(11) Melin J, Madanat-Harjuoja L, Heinävaara S, Malila N, Gissler M, Tiitinen A. Fertility treatments among female cancer survivors giving birth - a Finnish register-based study. Acta Oncol.2017 Aug; 56(8):1089-1093 (12) Leinonen MK, Miettinen J, Heikkinen S, Pitkäniemi J, Malila N. Quality measures of the populationbased Finnish cancer registry indicate sound data quality for solid malignant tumours. Eur J Cancer 2017; $77: 31-9$

(13) Steliarova-Foucher E, Stiller C, Lacour B. International classification of childhood cancer, third Edition.

Cancer 2005 Apr. 1; 103(7):1457-67

(14) Gissler M, Shelley J. Quality of data on subsequent events in a routine medical birth register. Medical Inform Internet Med. 2002 Mar; 27(1):33-8

(15) Gissler M, Klemetti R, Sevo'n T, Hemminki E. Monitoring of IVF birth outcomes in Finland: a data quality study. BMC Med Inform Decis Mak. 2004; 4:3

Wood S. Generalized Additive Models. 2006. New York: Chapman and Hall/CRC

(17) Luke B, Brown M, Missmer S, Spector LG, Leach RE, Williams M, Koch L, Smith YR, Stern JE, Ball GD, Schymura MJ. Assisted reproductive technology use and outcomes among women with a history of cancer. Hum Reprod 2016; 31: 183-189.

(18) Oktay K, Harvey BE, Partridge AH, Quinn GP, Reincke J, Taylor HS, Wallace WH, Wang ET, Loren AW. Fertility preservation in patients with cancer:ASCO Clinical practice guideline update. J Clin Oncol $2018 \mathrm{Jul}$; 36(19):1994-2001

(19) Peccatori FA, Azim HA Jr, Orecchia R, Hoekstra HJ, Pavlidis N, Kesic V, Pentheroudakis G. Cancer, pregnancy and fertility: ESMO Clinical practice guidelines for diagnosis, treatment and follow-up. Ann Oncol. 2013 Oct; 24(6):160-70 
(20) Lambertini M, Del Mastro L, Pescio M, Andersen CY, Azim HA, Peccatori FA, Costa M, Revelli A, Salvagno F, Gennari A, Ubaldi FM, La Sala GB, De stefano C, Wallace WH, Partridge AH, Anserini P. Cancer and fertility preservation: international recommendations from an expert meeting. BMC Med. 2016; 14:1 (21) Goldrat O, Kroman N, Peccatori F, Cordoba O, Pistilli B, Lidegaard O, Demeestere I, Azim HA. Pregnancy following breast cancer using assisted reproduction and its effect on long-term outcome. Eur J Cancer. 2015 Aug; 51(12):1490-6.

(22) Anderson RA, Wallace WHB. Antimullerian hormone, the assessment of the ovarian reserve, and the reproductive outcome of the young patient with cancer. Fert.Steril. 2013 May; 99(6):1469-75 (23) Freour T, Barriere P, Masson D. Anti-mullerian hormone levels and evolution in women of reproductive age with breast cancer treated with chemotherapy. Eur J Cancer 2017 Mar; 74:1-8 


\section{Table 2}

Number of events and adjusted incidence rate ratios (IRRs) for different fertility treatments between 1993 and 2012 among female cancer survivors compared to their siblings

\begin{tabular}{|c|c|c|c|c|}
\hline & & Any treatment & Ol & ART \\
\hline Status & & & & \\
\hline Survivors $\mathrm{N}=8,9$ & (\%) & $541(6.06)$ & $189(2.12)$ & $348(3.90)$ \\
\hline Siblings $N=9,49$ & & $358(3.77)$ & $219(2.31)$ & $141(1.48)$ \\
\hline & & & & \\
\hline Assuming no he & geneity in IR & ge and time perio & del MO) & \\
\hline Overall IRR $^{1}$ & & $1.43(1.25-1.65)$ & $0.83(0.68-1.02)$ & $2.41(1.97-2.96)$ \\
\hline Allowing hetero & eity in IRR by & ind period (model & & \\
\hline $\begin{array}{l}\text { Baseline IRR }^{1} \\
\text { (age 20-24, peri }\end{array}$ & $993-1997$ & $1.32(0.75-2.30)$ & $0.60(0.25-1.42)$ & $2.28(0.92-5.68)$ \\
\hline Relative excess & compared to & ine IRR & & \\
\hline Age at drug & $16-19$ & - & - & - \\
\hline purchase & $20-24$ & 1 & 1 & 1 \\
\hline & $25-29$ & $0.66(0.38-1.16)$ & $1.69(0.67-4.28)$ & $0.62(0.25-1.56)$ \\
\hline & $30-34$ & $0.86(0.48-1.54)$ & $1.64(0.67-4.04)$ & $0.41(0.17-0.97)$ \\
\hline & $35-41$ & $0.59(0.34-1.02)$ & $1.14(0.46-2.83)$ & $0.35(0.15-0.82)$ \\
\hline Test for heterog & & $p$-value $<0.001^{2}$ & $p$-value $=0.474^{2}$ & $p$-value $<0.001^{2}$ \\
\hline Period of drug & 1993-1997 & 1 & 1 & 1 \\
\hline purchase & 1998-2002 & $1.24(0.81-1.89)$ & $0.91(0.52-1.59)$ & $1.73(0.88-3.39)$ \\
\hline & 2003-2007 & $2.31(1.53-3.49)$ & $1.01(0.55-1.87)$ & 3.39 (1.79-6.41) \\
\hline & 2008-2012 & $1.73(1.18-2.53)$ & $0.89(0.53-1.50)$ & 2.65 (1.44-4.89) \\
\hline Test for heterc & & $p$-value $<0.001^{3}$ & $p$-value $=0.959^{3}$ & $p$-value $<0.001^{3}$ \\
\hline
\end{tabular}

IRR, incidence rate ratio; $\mathrm{Cl}$, confidence interval; OI, Ovulation induction; ART, Assisted renroductive technology

Bold indicates statistically significant incidence rate ratio

${ }^{1}$ Adjusted for calendar period and age at possible fertility drug purchase

Incidence rate of treatment was modelled using Poisson regression with following models for covariate effects: $M 0:$ age + period + status + age*period, $\mathrm{M1}$ : age + period + status + age * $^{*}$ eriod + age*status + period*status, M2: age + period + status + age*period + age*status, M3: age + period + status + age ${ }^{*}$ period + period*status.

${ }^{2} p$-value for comparing M1 vs M3 using likelihood ratio test ${ }^{3}$-value for comparing M1 vs M2 using likelihood ratio test 


\section{Table 3}

Incidence and conditional probability of ovulation induction treatments in cancer survivors and siblings according to age and time period of drug purchase

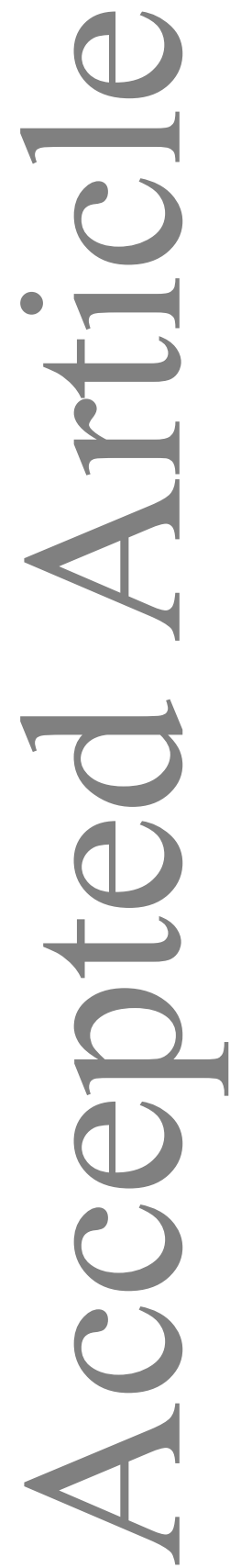




\begin{tabular}{|l|l|l|l|l|l|l|}
\hline & Total & 51 & 70 & 17944 & 17119 & \\
\hline
\end{tabular}

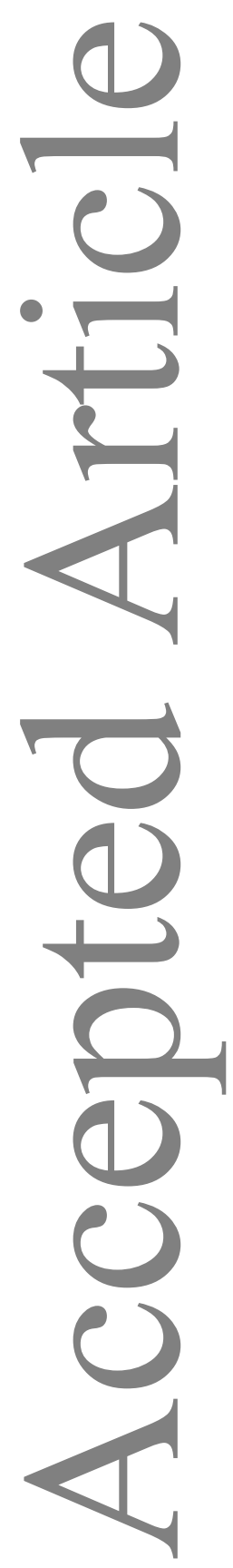

This article is protected by copyright. All rights reserved. 


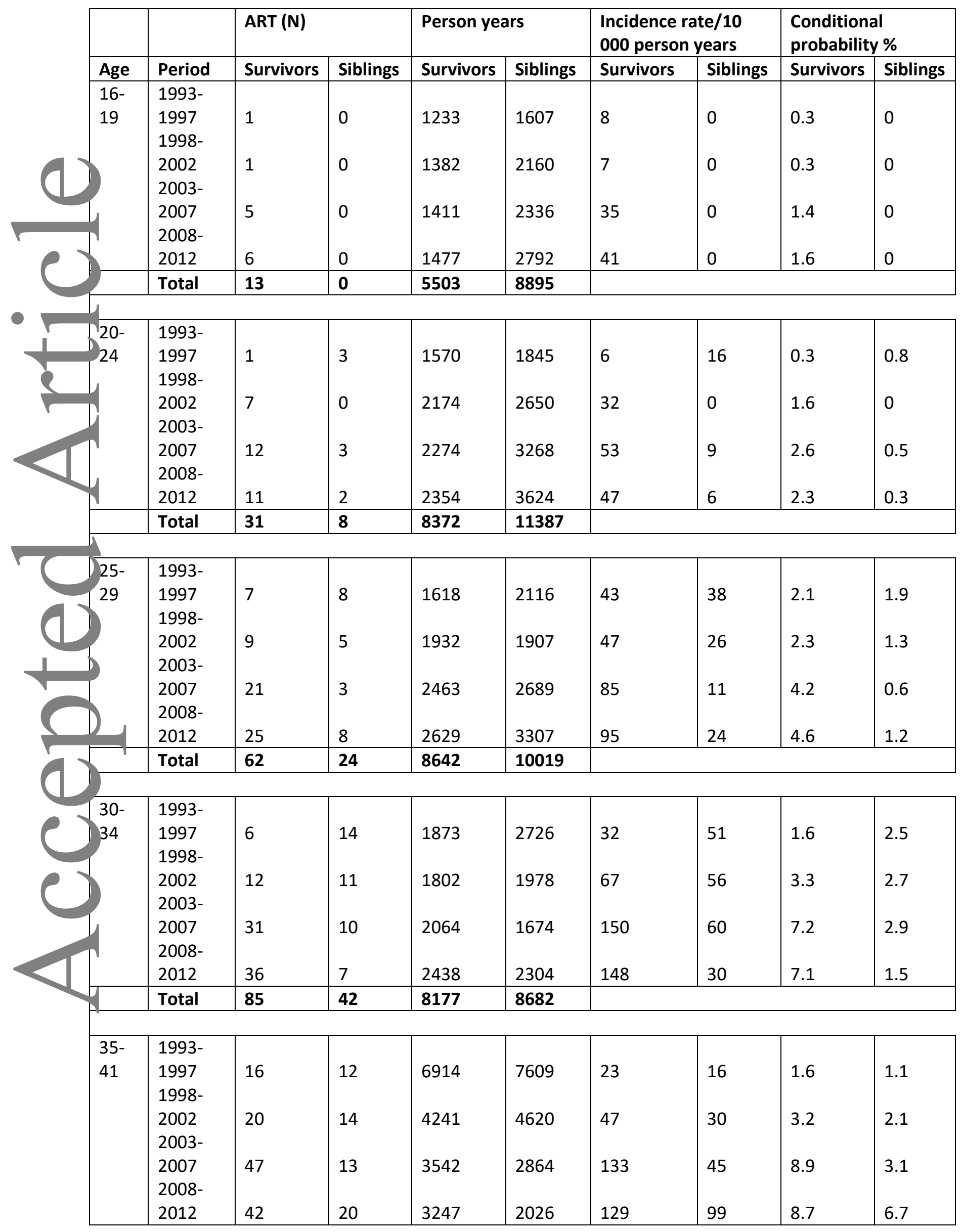

This article is protected by copyright. All rights reserved. 

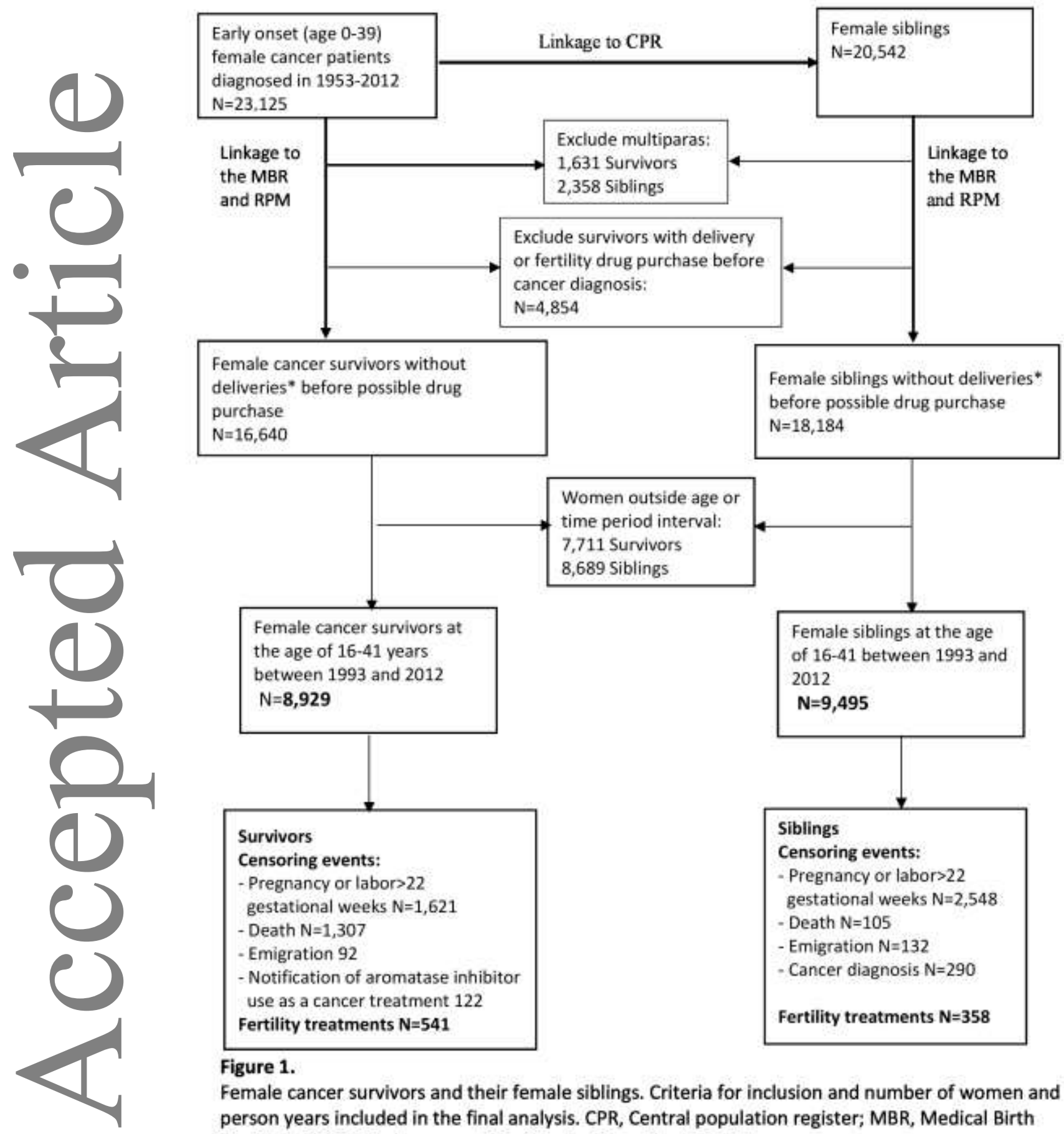

Figure 1.

Female cancer survivors and their female siblings. Criteria for inclusion and number of women and person years included in the final analysis. CPR, Central population register; MBR, Medical Birth Register; RPM, Reimbursement Register for Prescribed Medicines

*between 1987-2013 


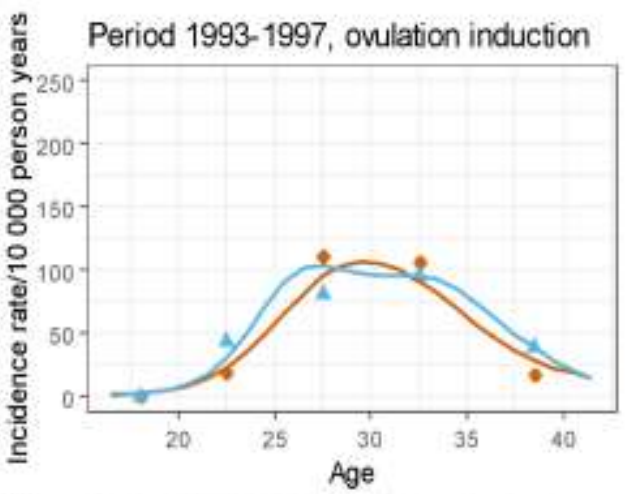

Period 1993-1997, ART

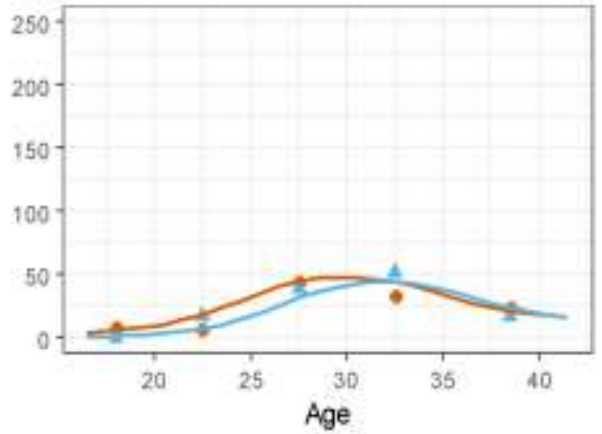

Period 1998-2002, ART

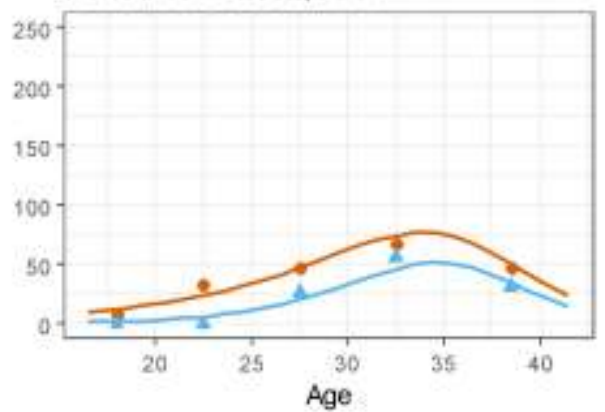

status

+ survivor

+ sibling

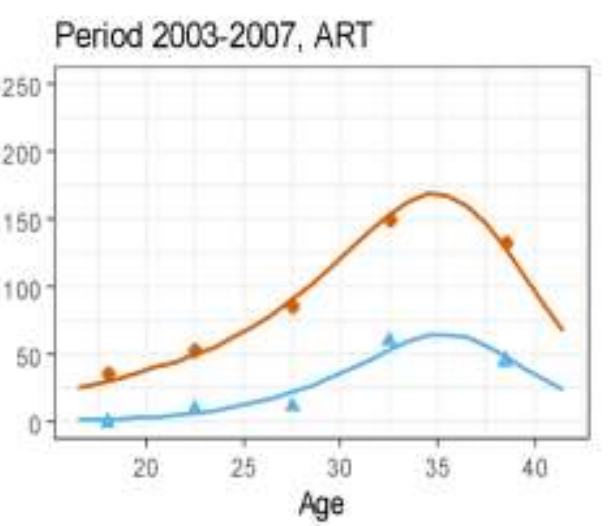

status

- survior

- sibling
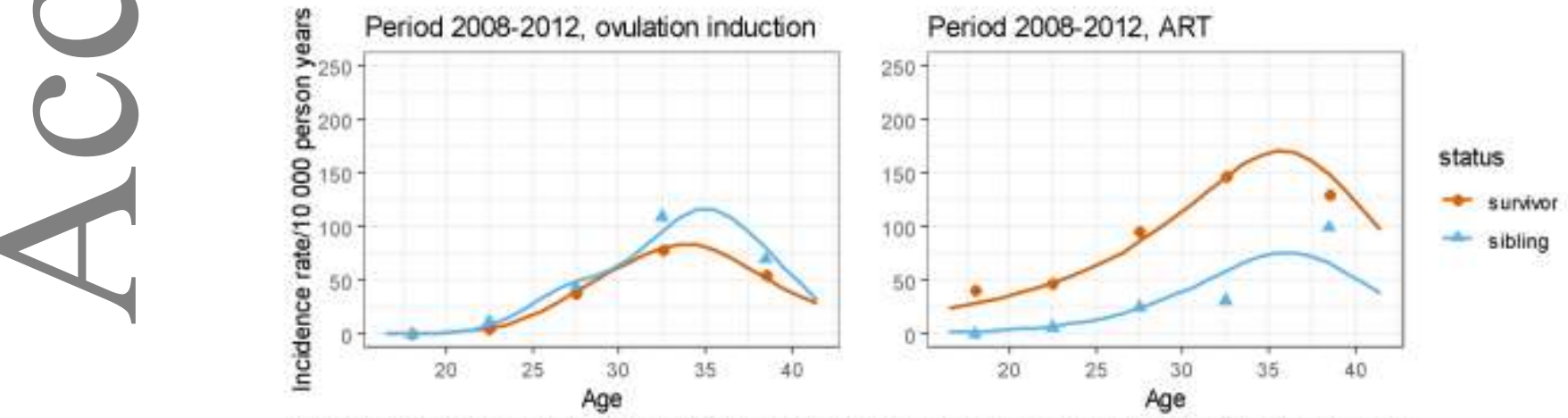

Figure 2. Incidence rates for ovulation induction and assisted reproductive technology (ART) in cancer survivors and siblings according to age at drug purchase in different time periods 


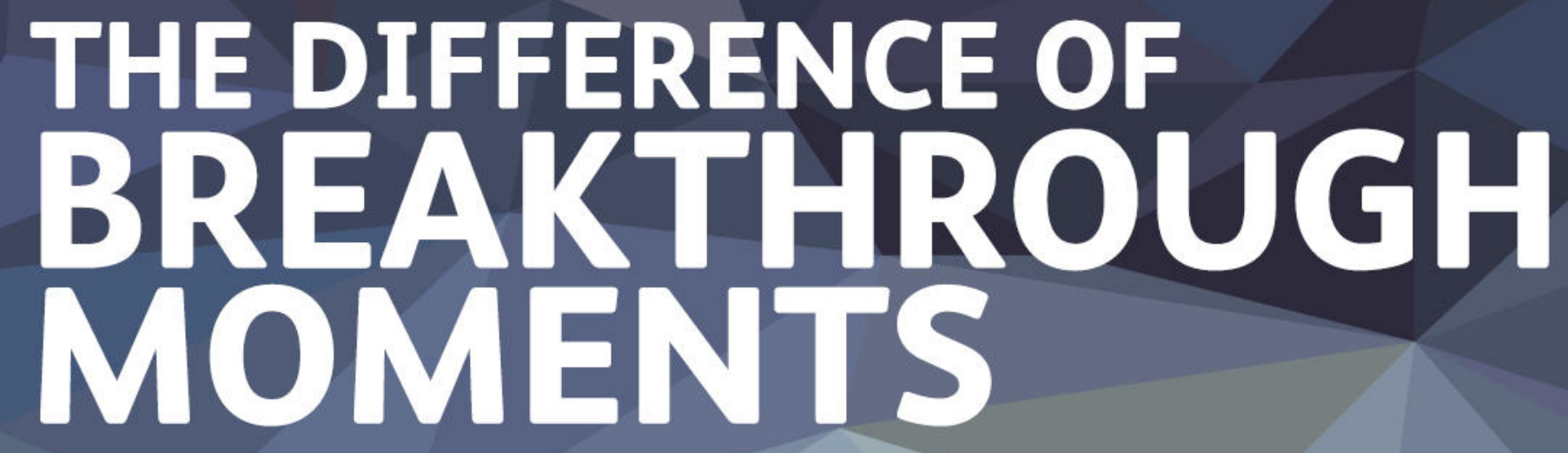

WITH AN INTEGRATED SOLUTION FOR

GROUNDBREAKING SINGLE-CELL RESEARCH

BD Accuri ${ }^{\text {TM }}$ C6 Plus Personal Flow Cytometer

BD FACSCelesta ${ }^{\text {TM }}$ Cell Analyzer

BD LSRFortessa ${ }^{\mathrm{TM}} \mathrm{X}$-20 Cell Analyzer

BD FACSMelody ${ }^{\text {TM }}$ Cell Sorter

Flow]o ${ }^{\text {TM }}$ Software

One of the largest portfolios of reagents

Discover more > 